\title{
Toward A Second Generation Electronic Nose at JPL: Sensing Film Optimization Studies
}

\author{
M. A. Ryan, M. L. Homer, H. Zhou, K. Manatt and A. Manfreda \\ Jet Propulsion Laboratory, California Institute of Technology
}

Copyright (c) 2001 Society of Automotive Engineers, Inc.

\begin{abstract}
Development of a second generation Electronic Nose at JPL is focusing on optimization of the sensing films to increase sensitivity and optimization of the array. Toward this goal, studies have focused on sources of noise in the films, alternatives to carbon black as conductive medium, measurement techniques, and development of an analytical approach to polymer selection to maximize the abilities of the array to distinguish among compounds.
\end{abstract}

\section{INTRODUCTION}

The JPL Electronic Nose (ENose) is a sensing array which is under development for environmental monitoring in crew habitat in spacecraft. The array is made up of weakly specific chemical sensors; the pattern and magnitude of response are used to identify and quantify the presence of contaminants. The device using the array is controlled and analyzed electronically, and mimics the action of the mammalian nose by recognizing patterns of response to vapors. Electronic noses such as this one are based on a biological model of "sniffing", detecting changes in the composition of the environment, and can be trained to detect new patterns.

The JPL Electronic Nose which flew on STS-95 used polymer/carbon composite films as sensing media in its 32 polymer/carbon composite conductometric sensors [13]. These sensors are conductometric chemical sensors which change resistance when the composition of the environment changes. The sensors are made by dispersing carbon black in polymer films, and the films are deposited over a set of electrodes. The polymer films swell or shrink in response to changes in the surrounding atmosphere. The change in film volume results in a change in conduction paths through the film, and is measured as a change in resistance across the film.

Review of the data from the STS-95 flight experiment led to a program to optimize the Electronic Nose. Optimization considerations include the sensing films, methods of data acquisition, and selection of polymers for films in the sensing array.

Sensing film optimization studies to increase sensitivity have been focused primarily on decreasing noise in the response. Studies with this goal have included investigation of the sensor films and of measurement methods. Studies of the films include studies of the polymers used in the films and of the fabrication methods, including consideration of several materials as possible replacements for carbon black as the conductive medium in the film, including noble metals, metal oxides and carbon nanotubes.

Studies of measurement techniques have investigated the use of $A C$ methods to follow sensor response. The first JPL ENose used DC measurements to monitor resistance changes in the sensing films. AC measurements of thin films can be more sensitive to changes at the interface of the electrodes and the films, and so AC techniques were considered for data acquisition.

The third goal in device optimization is improving analyte discrimination through a more analytical selection of polymers for the array. Previously, polymers were selected by statistical analysis of response of several tens of polymers to target gases [1,3]. Development of a more analytical approach has taken the form of developing a model of polymer-analyte interaction. The model of polymer-analyte interaction will be used in conjunction with data analysis algorithms to determine the best set of polymers as sensors for particular sets of analytes.

\section{NOISE STUDIES}

The magnitude of noise in the response of the sensor films varies widely, and so studies of the sources of noise were initiated. Responses from the sensors used in the Early Human Test Experiment [3] and from the sensors prepared as spares for those used in the Flight Experiment were analyzed to determine the possible sources of noise in the responses. 
Studies of the sources of noise in the polymer based sensors used in the JPL ENose have considered two primary sources - physical characteristics of the deposited film such as thickness and distribution of the conductive medium, and of the polymer itself such as melting point, molecular weight and glass transition temperature; and identity of the conductive medium. Initial evaluation of the electronics for data acquisition and device control showed that noise resulting from operating the electronics alone is at least one order of magnitude smaller than noise in the sensor film.

\section{FILM MORPHOLOGY, POLYMER IDENTITY AND RESISTANCE}

Sensor films were made by dissolving the selected polymer in solvent, dispersing the conductive medium, usually carbon black, in the solution, and depositing the solution on the sensor substrate [3,4]. The solution was deposited on each location in $1 \mu \mathrm{L}$ increments until the film was conductive. The target resistance for each film was $1000-50,000 \Omega$, but several sensors were outside that range.

In making sensor films, there is a tradeoff between film thickness and resistance. The thinner the film, the more sensitive it is likely to be, as lower concentrations of analyte are necessary to fill the thickness of the film and cause a substantial swelling which is monitored as resistance change. However, the thinner the film, the higher the resistance. Resistance in a thin film can be decreased by dispersing a greater quantity of conductive medium, but there is a limiting quantity beyond which the swelling in the polymer will be insufficient to cause a resistance change [5]. For this phase of ENose development, film thickness was not controlled in an effort to make films with resistance $1000-50,000 \Omega$. Identification of sources of noise will assist in finding approaches to keeping films as thin as possible for good sensitivity to low concentrations of analyte.

Because of the measuring circuit used in the JPL ENose, signals are amplified as the resistance of the film increases, and so noise will increase with resistance. This increase is systematic noise, and is not considered in these noise studies. Future generations of the device will use a circuit designed to minimize this effect.

Review of data taken on several films showed that there were several sensors which had similar baseline resistances but significantly different levels of noise in the response. These sensors were selected for further study. Electronic noise from the devices was measured to be about an order of magnitude smaller than noise in the sensors. Noise in the sensors was measured as normalized change in resistance, $\Delta R / R_{0}$ in clean air against a baseline resistance, $R_{0}$.
A review of physical characteristics of the polymers, i.e. molecular weight, glass transition temperature, and melting point, showed no correlation between those characteristics and the level of noise.

The most significant correlation to be found was in polymer identity. Films which had similar baseline resistance could have noise levels which differed by about an order of magnitude, yet this difference was reproducible from example to example of the films. Figure 1 shows a photograph of two sensors which had dramatically different noise levels. Several sensors made with poly vinylchloride/vinyl acetate consistently had baseline resistances of 30-40 $\mathrm{k} \Omega$ and noise on the order of $4 \times 10^{-5}$, while sensors made with polyvinyl acetate had baseline resistances in the same regime and noise on the order of $3 \times 10^{-4}$. In both cases, the films appear to have carbon homogeneously distributed.

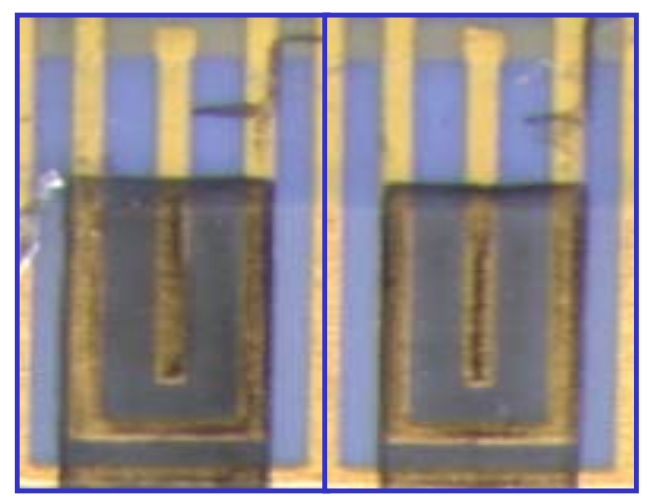

Figure 1. Sensors made with two different polymers, poly vinylchloride/vinyl acetate (left) and polyvinyl acetate (right) had similar baseline resistance but different levels of noise.

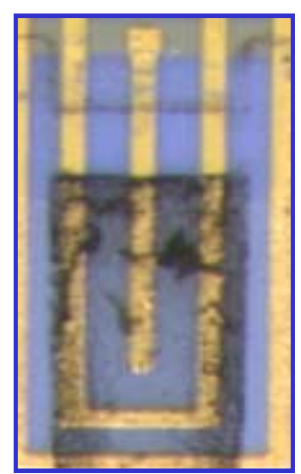

Figure 2. A sensor with poorly dispersed carbon black had low noise level.
Polymers which were poorly adherent such as vinyl alcohol/vinyl butyral copolymer tended to have high resistance in spite of deposition of several $\mu \mathrm{L}$ of solution. The high resistance was accompanied by high noise.

Even in cases where the carbon conductive medium was poorly dispersed in the films, as shown in Figure 2 for poly caprolactone, the noise level was consistent with the baseline resistance.

\section{MEASUREMENT TECHNIQUES}

Impedance measurement techniques were investigated to determine whether they would result in improved signal to noise ratios compared with DC measurements. It was hypothesized that a frequency which excluded 
some or all components of noise could be selected. Carbon-filled polyethylene oxide films were used to measure responses to methanol and water. Figure 3 shows the comparison between measurements at several frequencies as well as a DC measurement. The same film was used for each measurement. At 15 minutes, the film was exposed to $2500 \mathrm{ppm}$ of methanol, and at 30 minutes, the humidity is increased from $1 \% \mathrm{RH}$ to $30 \% \mathrm{RH}$. In the case of the DC measurement, the signal is expressed as the change in resistance divided by the initial resistance. In the case of the AC measurements, the signal is expressed as the change in the impedance divided by the initial impedance.

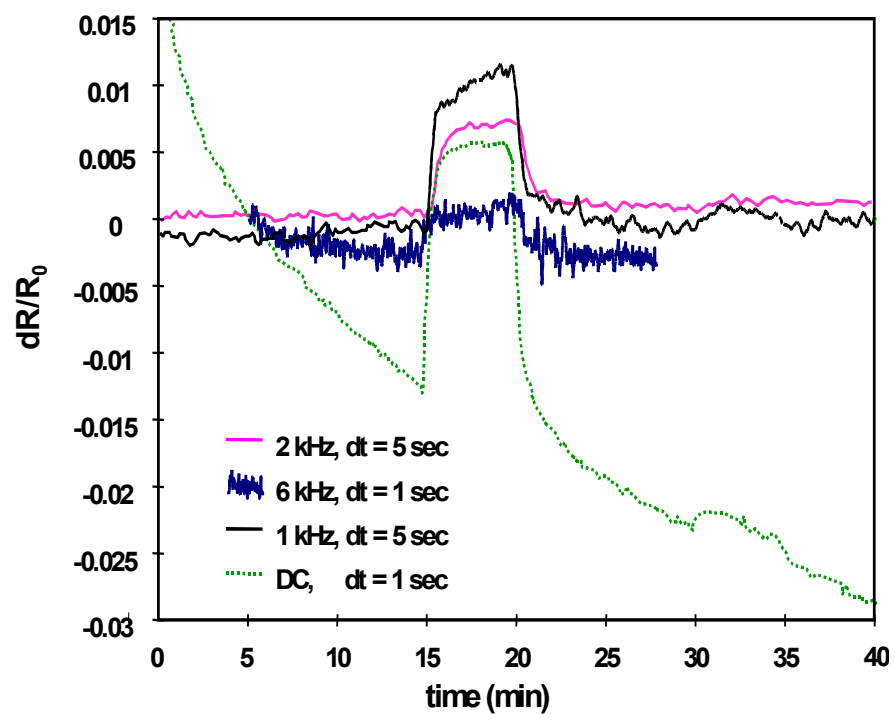

Figure 3: Response of a polymer/carbon composite sensing film at 3 $A C$ measuring frequencies and at $D C$.

Impedance was measured in the frequency range of 100$100,000 \mathrm{~Hz}$. In AC and DC measurements of the same event, sensor responses were comparable. As can be seen in Figure 3, it was possible to exclude some low frequency noise which contributed to baseline drift, but $\mathrm{AC}$ techniques did not yield better signal to noise ratios or increase sensor sensitivity significantly. It was determined that the best approach to increasing the signal to noise ratio would be to increase the number of averages on each DC measurement from 16 to 64 , which would add a few milliseconds to the time to query each sensor in the array and suppress some noise.

\section{CONDUCTIVE MEDIUM}

A number of investigators have studied the properties of polymer composites for sensor as well as other applications. [1-11] Although most of the work has been done using carbon-based fillers, there have also been studies of polymer composites which include metals [8] and vanadium oxide [6] as the conductive medium.

Currently, the conductive medium in the polymer composite sensor films is carbon black. A typical composite film is $20 \%$ carbon by weight $[1,4]$. It is possible that the carbon in the film contributes to analyte adsorption process in the sensor films, which could result in non-linear responses at low analyte concentration and in reaction between sorbed analyte and new analyte. In order to eliminate or reduce the possibility that carbon interferes with the sensing process, several alternative media were investigated.

Several materials have been used to make conductive films, including metals, metal oxides, and fullerenes [512]. A variety of materials were tested for use as conductive media in the polymer films, including gold powder (0.5-1.0 $\mu \mathrm{m})$, silver powder $(0.8-1.5 \mu \mathrm{m})$, nickel powder Inco type 123, vanadium oxides $\left(\mathrm{V}_{2} \mathrm{O}_{5}\right.$ and $\left.\mathrm{V}_{2} \mathrm{O}_{3}\right)$, $\mathrm{RuO}_{2}$, antimony doped $\mathrm{SnO}_{2}$ colloidal dispersion in water $(10 \mathrm{~nm})$, and single wall carbon nanotubes (SWNT). Single wall carbon nanotubes were donated by Prof. R. Hauge of Rice University; Ni powder was donated by Novamet. All other materials were purchased from Alfa Aesar. The materials were dispersed in several different polymers, primarily poly (ethylene oxide), poly (ethylenimine), poly (epichlorhydrin), and poly (4vinylphenol).

Of the metals and metal oxides, only silver resulted in a working sensor film. This film operated for only 20 hours until the silver particles oxidized and film resistance went above $2 \mathrm{M} \Omega$. In many cases, the metals and metal oxides could not be dispersed homogeneously in the polymer films. In other cases, where the metals dispersed, the baseline resistance was either greater than $2 \mathrm{M} \Omega$ or less than $50 \Omega$, neither of which is suitable for sensing. The percolation curve for the onset of conductance is very steep for these materials, and so it was not possible to find a load of metal which would result in a moderately conductive film.

SWNT are much more promising as conductive media for polymer-composite sensors. Although the SWNT are made of carbon and have vapor sorption properties similar to graphite [13], it is possible to make sensor films with a much smaller conductor/polymer ratio than with carbon black. Using poly (4-vinylphenol), two films were made for comparison, one with a carbon black load of $11 \%$ by weight and the other with a SWNT load of $3 \%$ by weight.

Figure 4 shows the response of each film to $100 \mathrm{ppm}$ of ethanol. It is possible to reduce the load of carbon in the film significantly and still get equivalent responses. Because SWNT will align spontaneously, it is possible that the tubes form conductive chains in the polymer film, making conductive pathways which are more direct than the pathways produced by the carbon particles. Thus, a smaller load of SWNT is required to make the film conductive in the $5-20 \mathrm{k} \Omega$ range. A carbon black load of $3 \%$ by weight would result in a film with a resistance in the $M \Omega$ range, and so would not be suitable for this device. 


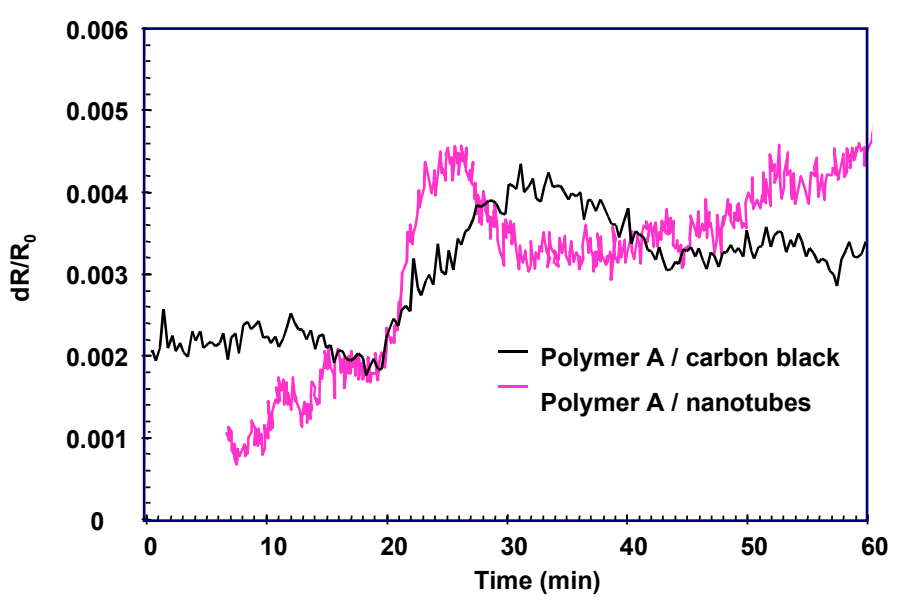

Figure 4. Sensor films with carbon black and single wall carbon nanotubes as the conductive medium respond to introduction of 100 ppm ethanol.

\section{POLYMER-ANALYTE INTERACTION}

A model of polymer-analyte interaction which takes account of physical and chemical processes in the sensor film in the presence and absence of analyte would assist in the process of selecting an array of polymer films. The concept fundamental to array based sensing is that the sensors are weakly specific and responses will form a pattern which is distinct enough for each analyte that the analyte can be identified. In the flight experiment phase of the JPL ENose program, polymers for the array were selected by analyzing response data for several tens of polymers [1,3] in order to find the set of 16 polymers which gave the most distinct patterns for the target analytes.

With a model of polymer analyte interaction, it will be possible to predict the response of a polymer to an analyte, which will allow selection of an array of polymer film sensors to match a set of potential analytes. In this way, sensors can be selected for changeout of the array as sensing needs change. If a validate model which takes account of functional group of the analyte can be developed, it may allow identification of unknown compounds by functional group as films with widely different responses are used in an array.

The first step toward development of such a model is investigation of polymers and analytes using the Linear Solvation Energy Relationship (LSER) method of M. H. Abraham [14], using published values measured or calculated for particular polymers and analytes [13, 15]. McGill et al. used these data to make predictions on SAW-polymer-sensor array responses to analytes at saturated vapor pressure [15]. More recently, Eastman et al. used solubility parameters to categorize chemiresistor responses [16].
In the LSER approach, polymer-analyte interaction is modeled as a solubility reaction where the analyte is the solute and the polymer the solvent. LSER describes solubility by combining solvation energy terms that quantify contributions from various solution processes, including polarizability, dipolarity, basicity, and acidity .

This approach predicts the response of a particular polymer to a particular analyte as a relative response is proportional to an equilibrium constant, $\mathrm{K}$, the ratio of the concentration of analyte in the vapor phase above the polymer film to the concentration of analyte sorbed within the polymer film. The LSER calculation of $\mathrm{K}$ is based on saturated vapor pressure of analyte and assumes equilibrium conditions.

Figure 5 shows the calculated equilibrium constant for six analytes in the presence of three polymer films. These equilibrium constants take account only of the sorption of analyte in the film. The relative response of a sensor film made from each polymer will be proportional to log $\mathrm{K}$ if the only cause of response is swelling in the film initiated by sorption of analyte vapor.

In Figure 5, analytes are ordered from largest to smallest dipole moment, from left to right. As can be seen in the figure, the response of a non-polar polymer such as polyisobutylene (PIB) is calculated to increase with decreasing polarity of the analyte, although the trend is not perfect. While benzene is less polar than toluene, $\mathrm{K}$ is larger for the solvation of benzene in PIB than for solvation of toluene.

Figure 6 compares the calculated relative responses of the three polymers to two analytes, benzene and 2propanol, alongside the measured response for a carbon-polymer composite sensing film. As can be seen, the LSER equilibrium constants are not exact predictors of the relative responses of the polymers to the analytes; however, the trends of the calculated responses match the trends of the measured responses. As a first step in developing a model, LSER is a useful tool, and can be used to select polymers for a set of analytes.

Solubility of analyte in the polymer film is only one aspect of film response; this first step in investigating solubility and sorption of the analyte does not account for adsorption of analyte on the carbon conductive medium dispersed in the film. Neither does the LSER approach account for analyte molecule diffusion within the film, film thickness, or hydration of the film.

Further work in constructing a model of polymer-analyte interaction with the goal of developing a method for predicting polymer film responses and selecting the best set of polymers for any one array is ongoing. 


\section{CONCLUSION}

Research on optimization of the sensing films and the sensing array in the JPL Electronic Nose is ongoing. Studies of sources of noise have found that the primary correlation for noise is polymer identity, a problem that can be solved by replacing noisy polymers. The circuit in the existing devices amplifies noise as a function of resistance; a new circuit is being designed to avoid that situation.

Studies of alternative conductive media have found that metals and metal oxides require too high a load to make films conductive to be useful in this device. The percolation curve for the onset of conductance in films is very steep for metals and metal oxides.

Single wall carbon nanotubes make a promising conductive medium for polymer composite conductometric sensors. The percolation curve for the onset of conductance is similar to that of carbon, yet a load of $3 \%$ by weight of nanotubes is sufficient to make a film conductive in the $5-50 \mathrm{k} \Omega$ range.

Optimization of the sensor array requires studies of the interaction between polymers used to make sensor films and target analytes. As a first step, solvation energy relationships have been used to describe the equilibrium condition for vapor sorbed in and above a polymer film. This approach offers a guide to film selection. Development of a model is ongoing, and the model will be expanded to include other processes such as analyte molecule diffusion within the film, film thickness, hydration of the film and possible reactions in the film.

\section{ACKNOWLEDGMENTS}

The research reported in this paper was carried out at the Jet Propulsion Laboratory, California Institute of Technology under a contract with the National Aeronautics and Space Administration. The authors thank Prof. Robert Hauge of Rice University for his donation of single wall carbon nanotubes.

\section{REFERENCES}

1. M. A. Ryan, M. L. Homer, H. Zhou, K. S. Manatt, V. S. Ryan and S. P. Jackson, "Operation of an Electronic Nose Aboard the Space Shuttle and Directions for Research for a Second Generation Device" Proc. $30^{\text {th }}$ International Conference on Environmental Systems, SAE (2000).

2. M.A. Ryan, M.L. Homer, M.G. Buehler, K.S. Manatt, B. Lau, D. Karmon and S. Jackson, "Monitoring Space Shuttle Air for Selected Contaminants Using an Electronic Nose," Proc. 28 $8^{\text {th }}$ International Conference on Environmental Systems, SAE (1998).
3. M.A. Ryan, M.L. Homer, M.G. Buehler, K.S. Manatt, F. Zee, and J. Graf, "Monitoring the Air Quality in a Closed Chamber Using an Electronic Nose," Proc. $27^{\text {h }}$ International Conference on Environmental Systems, SAE (1997).

4. M. C. Lonergan, E. J. Severin, B. J. Doleman, S. A. Beaber, R.H. Grubb, and N.S. Lewis, "Array-Based Vapor Sensing Using Chemically Sensitive, Carbon Black-Polymer Resistors," Chem. Mater., 8, 2298 (1996).

5. B Lundberg and B. Sundqvist, "Resistivity of a Composite Conducting Polymer as a Function of Temperature, Pressure, and Environment: Applications as a Pressure and Gas Concentration Transducer," J. Appl. Phys., 60, 1074 (1986).

6. K. A. Hu, D. Moffatt, J. Runt, A. Safari, and R. Newnham, " $\mathrm{V}_{2} \mathrm{O}_{3}$-polymer Composite Thermistors," J. Am. Ceram. Soc., 70, 583 (1987).

7. G. G. Neuburger and P.C. Warren, "Chemically Actuated Electronic Switch," Sens. Actuators B1, 326 (1990).

8. P. Bruschi and A. Nannini, "Low Temperature Behaviour of Ion-Beam-Grown Polymer-Metal Composite Thin Films," Thin Solid Films, 196, 201 (1991).

9. M.S. Freund and N.S. Lewis, "A Chemically Diverse Conducting Polymer Based Electronic Nose," Proc. National Academy of Science, 92, 2652, (1995).

10. A. Marquez, J. Uribe, R. Cruz, "Conductivity Variation Induced by Solvent Swelling of an Elastomer-Carbon Black-Graphite Composite," J. Appl. Polymer Sci., 66, 2221 (1997).

11. M. Hindermann-Bischoff, F. Ehrburger-Dolle, "Electrical Conductivity of Carbon Black-Polyethylene Composites: Experimental Evidence of the Change of Cluster Connectivity in the PTC Effect," Carbon, 39, 375 (2001).

12. J.W. Grate, M.H. Abraham, C.M. Du, R.A. McGill and W.J. Shuely, "Examination of Vapor Sorption by Fullerene, Fullerene-Coated SAWS, Graphite and Low-Polarity Polymers Using LSER," Langmuir, 11, 2125 (1995).

13. M. H. Abraham, G.J. Buist. P.L. Greillier, R.A. McGill, et al., "Solubility Properties in Polymers and Biological Media II. A New Method for the Characterisation of the Adsorption of Gases and Vapours on Solids," J. Chromatography, 409, 15 (1987).

14. R.A. McGill, M. H. Abraham and J.W. Grate, "Choosing Polymer Coatings for Chemical Sensors," Chemtech, 24, 27 (1994).

15. M. P. Eastman, R. C. Hughes, G. Yelton, A. J. Ricco, S. V. Patel, and M. W. Jenkins, "Application of the Solubility Parameter Concept to the Design of Chemiresistor Arrays," J. Electrochem. Soc., 146, 3907 (1999). 


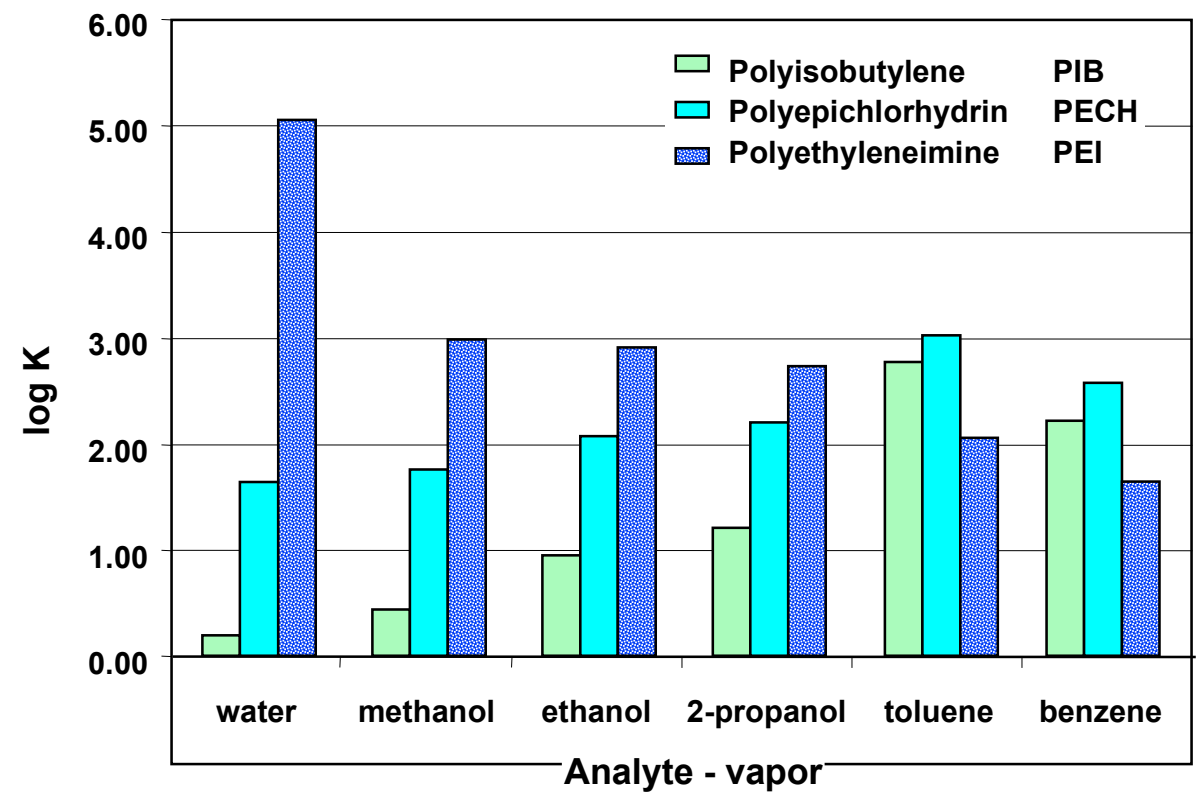

Figure 5: The log of the calculated equilibrium constant, $\mathrm{K}$, for equilibrium of saturated analyte vapor over a film of polymer. Log $\mathrm{K}$ should indicate the relative response of a sensor film made of the polymer, if the response is dependent only on swelling of the film initiated by sorption of analyte.

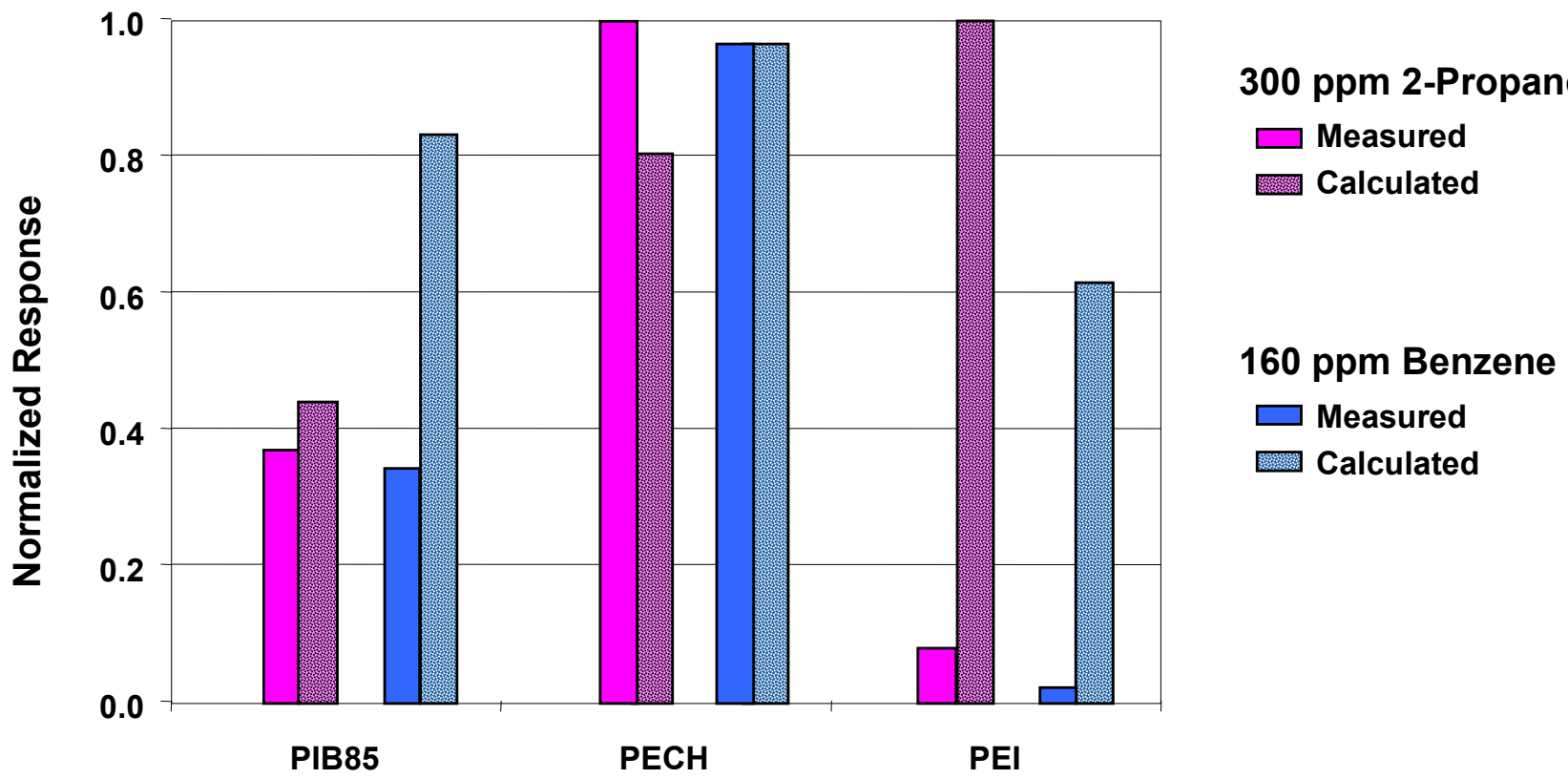

Figure 6: When compared with the calculated response, the measured response follows the same trends, but does not match the relative size of response. The LSER model does not take account of other processes in the response of the film. 